TITLE:

\title{
Impact of double-balloon rectal catheter use in external-beam radiotherapy for prostate cancer.
}

\author{
AUTHOR(S): \\ Takayama, Kenji; Mizowaki, Takashi; Negoro, \\ Yoshiharu; Norihisa, Yoshiki; Hiraoka, Masahiro
}

\section{CITATION:}

Takayama, Kenji ... [et al]. Impact of double-balloon rectal catheter use in external-beam radiotherapy for prostate cancer.. International journal of clinical oncology / Japan Society of Clinical Oncology 2011, 16(1): 50-56

\section{ISSUE DATE:}

2011-02

URL:

http://hdl.handle.net/2433/158361

\section{RIGHT:}

The final publication is available at www.springerlink.com; This is not the published version. Please cite only the published version.; この論文 は出版社版でありません。引用の際には出版社版をご確認ご利用くだ さい。 
Title: Impact of double-balloon rectal catheter use in external-beam radiotherapy for prostate cancer

\section{Kenji Takayama}

Takashi Mizowaki

Yoshiharu Negoro ${ }^{2}$

Yoshiki Norihisa

Masahiro Hiraoka

1

Department of Radiation Oncology and Image Applied Therapy, Graduate School of Medicine, Kyoto University

54 Kawahara-cho Shogoin Sakyo-ku, Kyoto, 606-8507, Japan

2

Department of Radiology, Tenri Hospital,

200 Mishima, Tenri-shi, Nara, 632-0015, Japan

Corresponding author: Takashi Mizowaki

Mailing address: $\quad$ Department of Radiation Oncology and Image Applied Therapy, Graduate School of Medicine, Kyoto University, 54 Kawahara-cho Shogoin Sakyo-ku, Kyoto, 606-8507, Japan

Phone: +81-75-751-3419

Fax: +81-75-771-9749

E-mail: mizo@kuhp.kyoto-u.ac.jp 


\section{Abstract}

Backgrounds: Prostate motion and rectal sparing are important issues in treatment strategy in external-beam radiotherapy for localized prostate cancer. To address these issues, we prospectively investigated the feasibility of using a double-balloon rectal catheter.

Methods: The rectal catheter has inner and outer balloons wedging the anus between them. Computed tomography (CT) examinations with and without the catheter were conducted in seven patients with localized prostate cancer treated by external-beam radiotherapy. Rectal wall sparing effect was evaluated using virtual three-dimensional conformal radiotherapy plans in each arm. To evaluate interfractional prostate motion, each patient underwent a series of four CT examinations consisting of a planning CT followed by three additional series of CT with and without a catheter during the course of radiotherapy.

Results: Virtual plans demonstrated the reduction of the dose to the rectum by expanding the posterior wall to outside the high- to intermediate-dose area when a catheter was applied. Interfractional prostate motion in the anteroposterior direction was effectively reduced by the catheter usage; The mean \pm standard deviation (SD) of the 
displacement in absolute value was $1.3 \pm 0.9 \mathrm{~mm}$ with a catheter as compared to $2.8 \pm 1.8$ mm without a catheter $(P=0.014)$, and the maximum displacement was successfully suppressed to $3 \mathrm{~mm}$ with a catheter compared to $6 \mathrm{~mm}$ without a catheter. Systematic and random components were also reduced with the catheter.

Conclusion: These results suggest the feasibility and clinical applicability of the double-balloon rectal catheter.

\section{Mini-Abstract}

The study demonstrated the feasibility of the double-balloon catheter use and its impact on rectal wall sparing effect and reduction of interfractional prostate motion in external-beam radiotherapy for prostate cancer. 


\section{(1) Introduction}

Prostate motion is an important issue in the application of external-beam radiotherapy for localized prostate cancer. The prostate often moves by several millimeters, and sometimes by more than $10 \mathrm{~mm}$, in the anteroposterior and superoinferior directions ${ }^{1-5}$. This motion should be taken into consideration when defining the planning target volume (PTV) from the clinical target volume (CTV) in treatment planning. An inappropriately wide margin will increase the dose to the rectum and the irradiated volume, resulting in a high incidence of rectal bleeding. Therefore, controlling prostate motion using a rectal balloon catheter is a promising approach ${ }^{6}$.

Inflation of the rectal balloon pushes the prostate anteriorly against the pubic symphysis, which restricts prostate motion. The balloon also has the potential to prevent exposure of a large part of the rectal wall to high or intermediate doses of radiation by expanding the posterior rectal wall away from the high-dose area.

Many studies ${ }^{6-15}$ have reported the advantages of rectal balloon catheters:

(a) Prostate immobilization (reduction of intrafractional prostate motion).

(b) Prostate repositioning (reduction of interfractional prostate motion).

(c) Reduction of rectal volume receiving high-dose radiation by rectal wall distension.

(d) Mild reduction of the dose to the anterior rectal wall due to a buildup effect. 
(e) Reduction of the rectal toxicity in clinical application.

(f) Visibility of the balloon in target localization using a lateral portal image, which enables adjustment of the posterior border of the prostate to the planned position.

Three types of balloon catheter differing in various characteristics, such as the shape of the balloon, hardness of the shaft, and handling performance, have been investigated independently at several institutes ${ }^{6-11}$. Each balloon catheter has advantages and disadvantages, and various results have been reported regarding the rectal sparing effect, prostate immobilization effect (intrafractional motion), and prostate position reproducibility (interfractional motion). van Lin et al. ${ }^{12}$ compared the rectal wall sparing effect between these three balloon catheters in each patient and reported that all types demonstrated a significant wall sparing effect in three-dimensional conformal radiotherapy (3D-CRT) as compared to no balloon and that two of the three types were superior to the remaining type. The volume of air for balloon inflation is also an important factor. Hille et al. ${ }^{15}$ compared rectal dose-volume histograms (DVHs) for different volumes of air and concluded that $60 \mathrm{~mL}$ was superior to $40 \mathrm{~mL}$. All of these reports were based on single-balloon catheters.

One of the problems when a single-balloon catheter is applied is balloon migration in the rectum, which reduces both reproducibility and stability of the prostate 
position. To address this problem, we investigated the feasibility of using a

double-balloon rectal catheter. In the present study, we evaluated the following two issues to prospectively examine impacts of the double-balloon catheter: reduction of dose to the rectum and irradiated volume (rectal wall sparing effect) and reduction of interfractional prostate motion during the course of radiotherapy.

\section{(2) Materials and methods}

\section{Double-balloon rectal catheter}

The rectal catheter used as an internal immobilization device for the prostate has two balloons as shown in Fig. 1. This is widely employed in the area of diagnostic radiology for barium enema procedures in Japan (Create Medic Co., Ltd., Yokohama, Japan) ${ }^{16}$. Both the shaft and the balloons were made of styrene elastomer, and the inner and outer balloons were inflated with $40 \mathrm{~mL}$ and $25 \mathrm{~mL}$ of air, respectively, in the present study. The anus was wedged between the inner and outer balloons, which is useful in repositioning and immobilizing the balloons. In addition, the inner balloon pushes the prostate against the pubic symphysis. The catheter shaft had a diameter of $11.9 \mathrm{~mm}$, and the dimensions of the inner balloon, filled with $40 \mathrm{~mL}$ of air, were $50 \mathrm{~mm}$ in diameter and $30 \mathrm{~mm}$ in length. Figure 2 shows computed tomography (CT) images of a patient with the catheter 
fixed in the rectum.

One catheter was reused for one patient after cleaning to minimize additional cost. A leakage test was always performed immediately before use. If air leakage was detected, a new catheter was applied. Xylocaine jelly was used for lubrication when the catheter was inserted into the rectum.

\section{$\underline{\text { Patient characteristics }}$}

The study population consisted of seven patients with localized prostate cancer treated by external-beam radiotherapy between August 2000 and June 2002. All subjects gave their informed consent to participation in this study. The patients were treated with 3D-CRT or intensity-modulated radiation therapy (IMRT) with curative intent. Prior to radiotherapy, all of the subjects were treated with neoadjuvant maximum androgen blockade therapy. Patients with difficulties in positioning, such as those with paralysis of the lower extremities, were excluded from the study.

\section{(i) Evaluation of an effect in rectal wall sparing}

To evaluate the impact of the balloon catheter in the rectal wall sparing effect, CT examinations with and without the balloon catheter were conducted in all patients. Virtual 
3D-CRT plans were designed with both CT study sets for each patient using a 3D

radiation treatment planning system (3D-RTPS) (CadPlan ver. 6.1.5; Varian Associates, Palo Alto, CA). The plans were coplanar six-field 3D treatment plans, and the PTV was defined as the prostate plus a 3D margin of $10 \mathrm{~mm}$ except for the posterior border, where a 6-mm margin was applied. The edge of the multileaf collimator was placed $3 \mathrm{~mm}$ outside the border of the PTV to account for beam penumbra. The prescription dose was 70 Gy in 35 fractions. The rectal wall was delineated from $5 \mathrm{~mm}$ above to $5 \mathrm{~mm}$ below the upper and lower limits of the PTV, respectively.

Comparisons between plans with and without an inflated balloon catheter were made regarding the rectal wall, prostate, and PTV based on the DVH. The differences in the percent volumes of the rectal wall receiving 35, 50, and $63 \mathrm{~Gy}\left(\mathrm{~V}_{35}, \mathrm{~V}_{50}\right.$, and $\mathrm{V}_{63}$, respectively) were evaluated with a paired $t$-test.

\section{(ii) Effect on interfractional prostate motion}

Each patient underwent a series of four CT examinations consisting of a planning CT scan (P-CT) followed by three additional series of CT with and without a catheter during the course of radiotherapy. The prostate was delineated in all CT series by one physician to eliminate interobserver variation. For each patient, the setup error was measured on CT 
images based on pelvic bony structures by comparing between P-CT and each additional CT series. The position of the center of the prostate (COP) on each CT series was determined, and the data were used for compensation of the setup error. The displacements of the positions of COPs on the three additional CT series from the corresponding COP on the P-CT were calculated as the interfractional prostate motion. They were calculated in the anteroposterior (A-P), superoinferior (S-I), left-right (L-R), and 3D $\left\{(3 \mathrm{D}\}=\left((\mathrm{A}-\mathrm{P})^{2}+(\mathrm{S}-\mathrm{I})^{2}+(\mathrm{L}-\mathrm{R})^{2}\right)^{1 / 2}\right\}$ directions. Seven paired prostate motion data with and without a catheter were analyzed using Wilcoxon’s matched-pairs signed-rank test to compare the differences in mean absolute values of COP displacement with and without the balloon catheter. Systematic and random components of the interfractional prostate motion were also analyzed with and without the balloon.

All measurements were performed with 3D-RTPS as described above. No image registration tool was used because no reliable tool was available when the study was conducted. CT image resolutions were $256 \times 256$ with a field of view (FOV) size of 30 $\mathrm{cm}$. Each calculated value was rounded off to an integer because of the resolution.

\section{(3) Results}

All of the patients included in this study tolerated the use of a rectal balloon catheter at a 
series of four CT examinations.

\section{(i) Rectal wall sparing effect}

DVHs of the rectal wall and the PTV for all patients are shown in Fig. 3. Table 1 presents the mean ( \pm 1 standard deviation: $S D$ ) of $V_{35}, V_{50}$, and $V_{63}$ of the rectal wall with and without a balloon catheter. When a rectal balloon catheter was applied, the percent volumes of the rectal wall receiving both high and intermediate doses were significantly reduced without compromising the PTV coverage. Figure 4 shows the two-dimensional dose distribution on axial and sagittal images. The inner balloon expands the rectum at the level of the prostate where the posterior rectal wall comes close to the prostate due to the puborectalis muscle under natural conditions without the balloon catheter.

Therefore, the rectal balloon catheter could prevent inclusion of the posterior rectal wall within the intermediate-dose area.

\section{(ii) Interfractional prostate motion}

The absolute values of COP displacement are shown in Table 2. Application of a catheter significantly reduced COP displacements in the A-P and 3D directions in comparison with the values without a catheter. The mean \pm SD displacement in the A-P 
direction was $1.3 \pm 0.9 \mathrm{~mm}$ with a catheter as compared to $2.8 \pm 1.8 \mathrm{~mm}$ without a catheter $(P=0.014)$, and the maximum displacement was successfully suppressed to 3 $\mathrm{mm}$ with a catheter compared to $6 \mathrm{~mm}$ without a catheter. In contrast, no significant differences with and without the balloon catheter were observed with respect to displacements in the S-I and L-R directions, although displacement in the S-I direction tended to be smaller with a catheter.

Table 3 shows the results of analyzing the systematic and random displacements. In the A-P direction, both systematic and random displacements were smaller with the balloon catheter; the mean \pm SD of the systematic displacements was $0.4 \pm 1.3 \mathrm{~mm}$ and the SD of random components was $0.9 \mathrm{~mm}$ with a catheter, as compared to $1.4 \pm 2.4$ $\mathrm{mm}$ and $1.9 \mathrm{~mm}$, respectively, without a catheter. In the S-I direction, only the systematic components were smaller with the balloon catheter, and the random components were almost the same with and without the catheter. Both components in the L-R direction were similar with and without the balloon catheter.

\section{(4) Discussion}

This feasibility study demonstrated the utility of the original rectal catheter with double balloons in comparison to treatment without the catheter with regard to the rectal wall 
sparing effect on 3D-CRT and the reduction of interfractional prostate motion in the A-P direction. Note that systematic and maximum interfractional motions were reduced because systematic components have greater impact on the PTV margin than random components, and the small maximum motion guarantees CTV dose coverage in all fractions. In terms of the dose to the rectum, the posterior wall was expanded by the inner balloon to outside the high- to intermediate-dose area. In even an IMRT plan, the posterior rectal wall can be spared when a balloon catheter is applied versus no catheter because of the advantage in the long distance between the prostate and the posterior rectal wall.

To compare our double-balloon catheter with other single-balloon catheters reported to date, we present a review of three types of single-balloon catheter that have been investigated for their advantages in the clinical application at other institutes. van Lin et al. ${ }^{12}$ numbered each endorectal balloon (ERB) as follows in their paper in which they compared the rectal wall sparing effects among these three balloon catheters in the same patients.

ERB1: a flexible rectal balloon catheter made of soft rubber, commonly used in diagnostic radiology for barium enema procedures (Nordmann, Rüsch AG, Kernen, Germany). The silicon-latex balloon is $55 \mathrm{~mm}$ in length and $40 \mathrm{~mm}$ in diameter when 
filled with $40 \mathrm{~mL}$ of air. Gerstner et al. ${ }^{7}$ and Wachter et al. ${ }^{8}$ used this type of balloon.

ERB2: a catheter originally designed as an endorectal coil for magnetic resonance imaging (Medrad, Pittsburgh, PA). The latex balloon has a specific anatomical concave shape to conform to the prostate-rectal interface. This has a length of $90 \mathrm{~mm}$ and a diameter of $45 \mathrm{~mm}$ when inflated with $80 \mathrm{~mL}$ of air, and is the largest balloon among these three catheters. D’Amico et al. ${ }^{9}$ and van Lin et al. ${ }^{12,13}$ reported the results of this usage.

ERB3: a catheter applied by Teh et al. ${ }^{6}$ and McGary et al. ${ }^{10}$ at Baylor College, consisting of a non-latex retention cuff on a rigid 15-cm shaft (E-Z-EM, Westbury, NY). Inflated with $100 \mathrm{~mL}$ of air, the length of the balloon is $45 \mathrm{~mm}$ and the diameter is $60 \mathrm{~mm}$.

Compared to these single-balloon catheters, the most advantageous feature of our double-balloon catheter is positioning stability. The wedging of the anal canal between the inner and outer balloons enables reproduction of the prostate position in all sessions and fixing of the position during a session.

With regard to interfractional prostate motion, Gerstner et al. ${ }^{7}$ and Wachter et al. ${ }^{8}$ reported the significant effect of a balloon (ERB1) on the reduction of the maximum prostate displacement in the A-P direction in their study of 10 patients; the maximum displacement ranged from 0 to $6 \mathrm{~mm}$ (mean: $3.6 \mathrm{~mm}$ ) with the balloon and from 1 to 17 
mm (mean: $7.4 \mathrm{~mm}$ ) without the balloon. These results were similar to those of the present study when the differences in measurement point are taken into account (posterior prostate border vs. center of the prostate). In contrast, McGary et al. ${ }^{10}$, who used ERB3 inflated with $100 \mathrm{~mL}$ of air, reported that the interfractional prostate motion was consistently less than $1 \mathrm{~mm}$ in the A-P direction and that the intrafractional motion in normal breathing was negligible in 10 patients with gold seed implants. The air volume of $100 \mathrm{~mL}$, which inflates the balloon to the largest diameter of $60 \mathrm{~mm}$, seems to enable such a high degree of prostate motion reduction. In the comparison test by van Lin et al. ${ }^{12}, 5$ of 20 patients experienced pain and local high pressure on the rectal wall, although this feeling disappeared within 1 min. Patel et al. ${ }^{11}$ also used ERB3 but the balloon was inflated with $60 \mathrm{~mL}$ of air, and the mean SD of the balloon position variability relative to the pelvis was $2.6 \mathrm{~mm}$ in the A-P direction in five weekly port films. These reports indicated that the air volume should be decided depending on the balance between the expected advantage (motion reduction) and the tolerable disadvantage (patient discomfort).

We considered a volume of $40 \mathrm{~mL}$ of air in the inner balloon of our catheter to be appropriate, and this was supported by the results of the present study regarding interfractional prostate motion, the rectal sparing effect, and patient tolerance. Excessive 
air volume would make wedging the anus between the double balloons difficult. In addition, product safety reasons exist as the double-balloon catheter product description states that the air volume for the inner balloon should be limited to $50 \mathrm{~mL}$.

Our catheter is made of soft material (styrene elastomer), which provides flexibility and improves patient tolerance. In addition, the catheter shaft is sufficiently thick to maintain consistency in its shape even if compressed by the anal canal and rectum. This makes the insertion easy and smooth, and also contributes to positional consistency in the rectum during treatment. Our catheter is an open system, which means that the catheter tip has side holes that enable excretion of stool and gas in the rectum. ERB1 and ERB3 are also open systems because they were made for barium study, whereas ERB2 is a closed system because it was designed as an endorectal coil for magnetic resonance imaging. In a comparative study of interfractional prostate position variation with or without ERB2, van Lin et al. ${ }^{12}$ reported that the presence of gas and stool beside the ERB can explain why the random interfractional displacements were not reduced by the ERB and remained almost unchanged in all three directions. Therefore, we concluded that an open system is more suitable than a closed system for reproducibility of balloon position and shape in the rectum.

In clinical application, most physicians may be worried that patient discomfort may 
compromise tolerance to rectal balloon usage. We were also concerned about patient tolerance at the beginning of the study. However, all of the patients included in this study tolerated the treatment well. Results have been reported regarding patient tolerance at various institutes where rectal balloon catheters are used routinely as prostate immobilization devices. Wachter et al. ${ }^{8}$ reported that 104 of 108 patients (96.3\%) tolerated the usage of a rectal balloon up to the end of the treatment course, while balloon application had to be stopped in 4 patients (3.7\%) because of proctitis symptoms and it could not be applied in 1 patient because of proctological disease. McGary et al. ${ }^{10}$ in the Baylor College group also reported tolerance in their initial experience among over 400 patients treated with IMRT using the rectal balloon.

Some studies also reported the advantages of visibility of the balloon in target localization using lateral portal images, which enables adjustment of the posterior border of the prostate to the planned position. A combination of a rectal balloon and image-guided radiation therapy (IGRT) technique can make the best advantage of prostate immobilization and target localization because it enables both daily prostate position correction and prostate immobilization. This combination may aid in dose escalation or hypofractionation studies to treat prostate cancer with a tighter PTV margin than is possible with conventional treatment. 
In conclusion, we demonstrated the impact of the double-balloon rectal catheter in reducing the radiation dose to the rectum in 3D-CRT. We also demonstrated the impact on reducing the interfractional prostate motion, especially systematic and maximum displacement in the A-P direction. These results suggest the feasibility and clinical applicability of the double-balloon rectal catheter. 


\section{(5) Conflict of Interest Statement}

None of the authors have any financial relationship with any commercial entity that has an interest in the subject matter or materials discussed in this manuscript.

\section{(6) Acknowledgments}

This work was partially supported by Grants-in-Aid for scientific research from the Ministry of Education, Culture, Sports, Science, and Technology (20229009) of Japan; Grants-in-Aid for Scientific Research on Priority Areas Cancer from the Ministry of Education, Culture, Sports, Science, and Technology (17016036) of Japan. These study sponsors played no role in the study design, collection, analysis and interpretation of data, writing of this manuscript, or the decision to submit this manuscript for publication.

This work was partially presented at the 44th Annual Meeting of American Society for Therapeutic Radiology and Oncology, October 2002, New Orleans, LA.

The English in this document has been checked by at least two professional editors, both native speakers of English. For a certificate, please see: 
http://www.textcheck.com/certificate/jnhRXT 


\section{References}

1. Roeske JC, Forman JD, Mesina CF, et al. (1995) Evaluation of changes in the size and location of the prostate, seminal vesicles, bladder, and rectum during a course of external beam radiation therapy. Int J Radiat Oncol Biol Phys 33:1321-1329

2. Antolak JA, Rosen, II, Childress CH, et al. (1998) Prostate target volume variations during a course of radiotherapy. Int J Radiat Oncol Biol Phys 42:661-672

3. Stroom JC, Koper PC, Korevaar GA, et al. (1999) Internal organ motion in prostate cancer patients treated in prone and supine treatment position. Radiother Oncol 51:237-248

4. Zelefsky MJ, Crean D, Mageras GS, et al. (1999) Quantification and predictors of prostate position variability in 50 patients evaluated with multiple CT scans during conformal radiotherapy. Radiother Oncol 50:225-234

5. $\quad$ Langen KM, Jones DT (2001) Organ motion and its management. Int J Radiat Oncol Biol Phys 50:265-278

6. Teh BS, Woo SY, Butler EB (1999) Intensity modulated radiation therapy 
(IMRT): a new promising technology in radiation oncology. Oncologist $4: 433-442$

7. Gerstner N, Wachter S, Dorner D, et al. (1999) [Significance of a rectal balloon as internal immobilization device in conformal radiotherapy of prostatic carcinoma]. Strahlenther Onkol 175:232-238

8. Wachter S, Gerstner N, Dorner D, et al. (2002) The influence of a rectal balloon tube as internal immobilization device on variations of volumes and dose-volume histograms during treatment course of conformal radiotherapy for prostate cancer. Int J Radiat Oncol Biol Phys 52:91-100

9. D'Amico AV, Manola J, Loffredo M, et al. (2001) A practical method to achieve prostate gland immobilization and target verification for daily treatment. Int J Radiat Oncol Biol Phys 51:1431-1436

10. McGary JE, Teh BS, Butler EB, et al. (2002) Prostate immobilization using a rectal balloon. J Appl Clin Med Phys 3:6-11

11. Patel RR, Orton N, Tome WA, et al. (2003) Rectal dose sparing with a balloon catheter and ultrasound localization in conformal radiation therapy for prostate cancer. Radiother Oncol 67:285-294

12. van Lin EN, Hoffmann AL, van Kollenburg P, et al. (2005) Rectal wall sparing 
effect of three different endorectal balloons in 3D conformal and IMRT prostate radiotherapy. Int J Radiat Oncol Biol Phys 63:565-576

13. van Lin EN, van der Vight LP, Witjes JA, et al. (2005) The effect of an endorectal balloon and off-line correction on the interfraction systematic and random prostate position variations: a comparative study. Int J Radiat Oncol Biol Phys 61:278-288

14. van Lin EN, Kristinsson J, Philippens ME, et al. (2007) Reduced late rectal mucosal changes after prostate three-dimensional conformal radiotherapy with endorectal balloon as observed in repeated endoscopy. Int J Radiat Oncol Biol Phys 67:799-811

15. Hille A, Schmidberger H, Tows N, et al. (2005) The Impact of Varying Volumes in Rectal Balloons on Rectal Dose Sparing in Conformal Radiation Therapy of Prostate Cancer A Prospective Three-Dimensional Analysis. Strahlenther Onkol 181:709-716

16. http://www.createmedic.co.jp/english/index.html 


\section{Figure legends}

Figure 1: A rectal catheter with double balloons

Used as an internal immobilization device for the prostate. The inner balloon was inflated with $40 \mathrm{ml}$ of air and outer one with $25 \mathrm{ml}$ of air in our study. The catheter is widely employed in the area of diagnostic radiology for barium enema procedures.

Figure 2: CT images of a patient with a double balloon catheter in a prone position 2.a An axial image in the level of prostate. The inner balloon pushes the prostate against the pubic symphysis.

2.b A median sagittal image. The anus is wedged between the inner and the outer balloon.

Figure 3: DVHs for the rectal wall and PTV

3.a: DVHs for the rectal wall, 3.b: DVHs for PTV

Each thin line shows a DVH of each patient. In the figure 3.a, each thick line shows the mean DVH of each group, and bars indicate 1 standard deviation of each group at the representative dose levels.

Abbreviation: DVH: dose volume histogram

Figure 4: Dose distribution with or without a rectal balloon catheter 
The inner balloon expands the rectum and prevents the posterior rectal wall

from being included within the intermediate-dose area. 


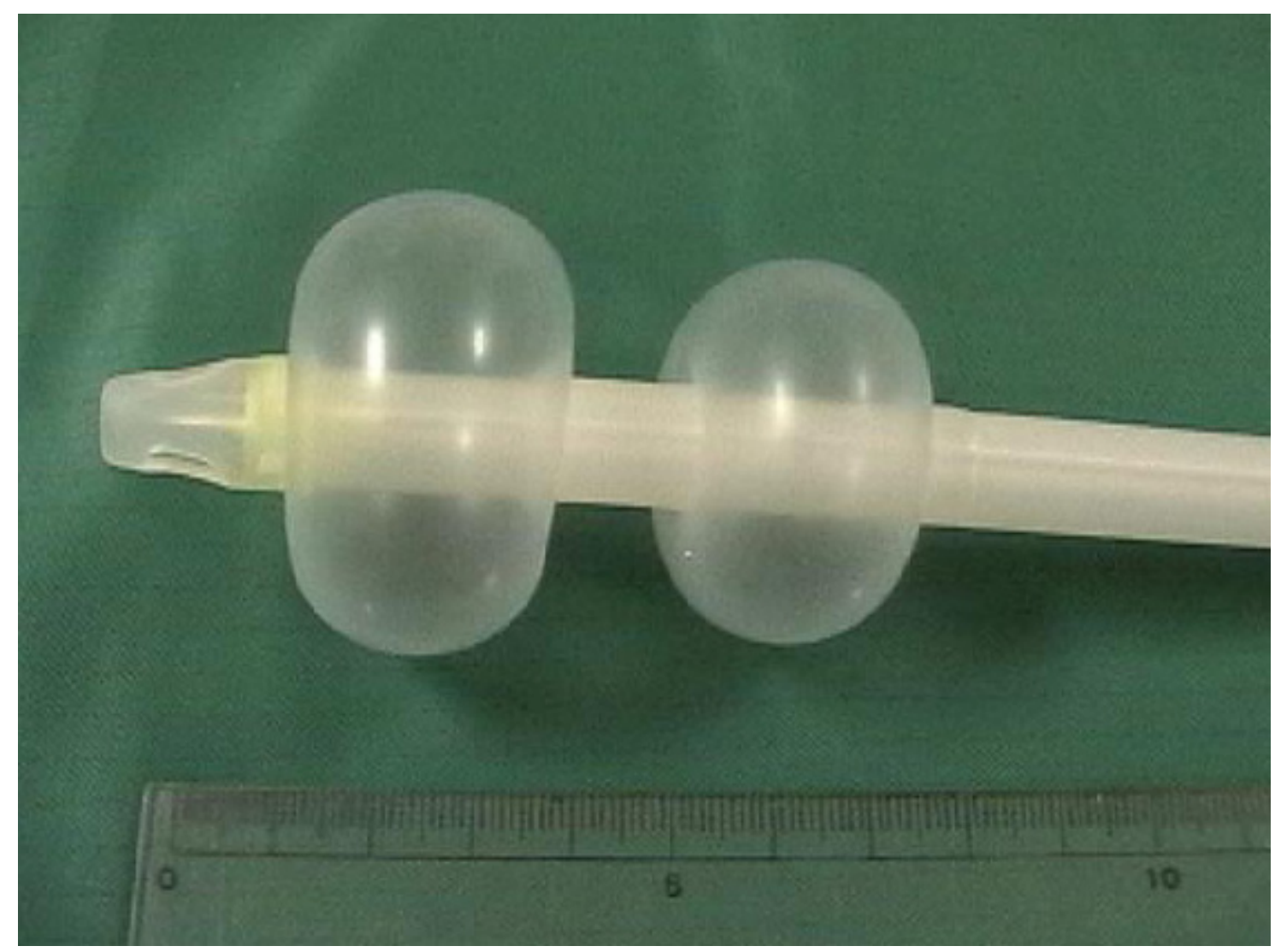

Figure 1: A rectal catheter with double balloons

Used as an internal immobilization device for the prostate. The inner balloon was inflated with $40 \mathrm{ml}$ of air and outer one with $25 \mathrm{ml}$ of air in our study.

The catheter is widely employed in the area of diagnostic radiology for barium enema procedures. 


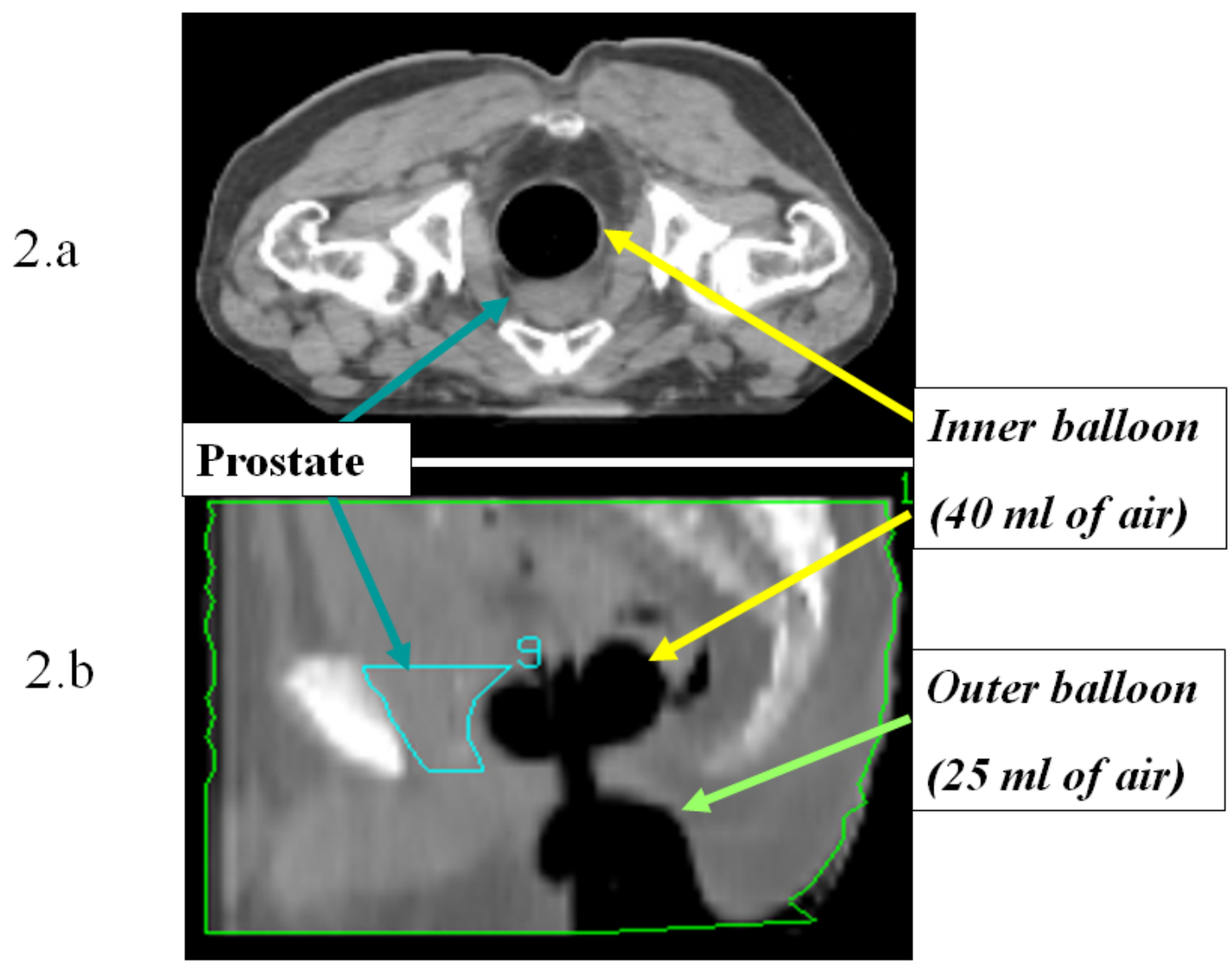

Figure 2: CT images of a patient with a double balloon catheter in a prone position

2.a An axial image in the level of prostate. The inner balloon pushes the prostate against the pubic symphysis.

2.b A median sagittal image. The anus is wedged between the inner and the outer balloon. 


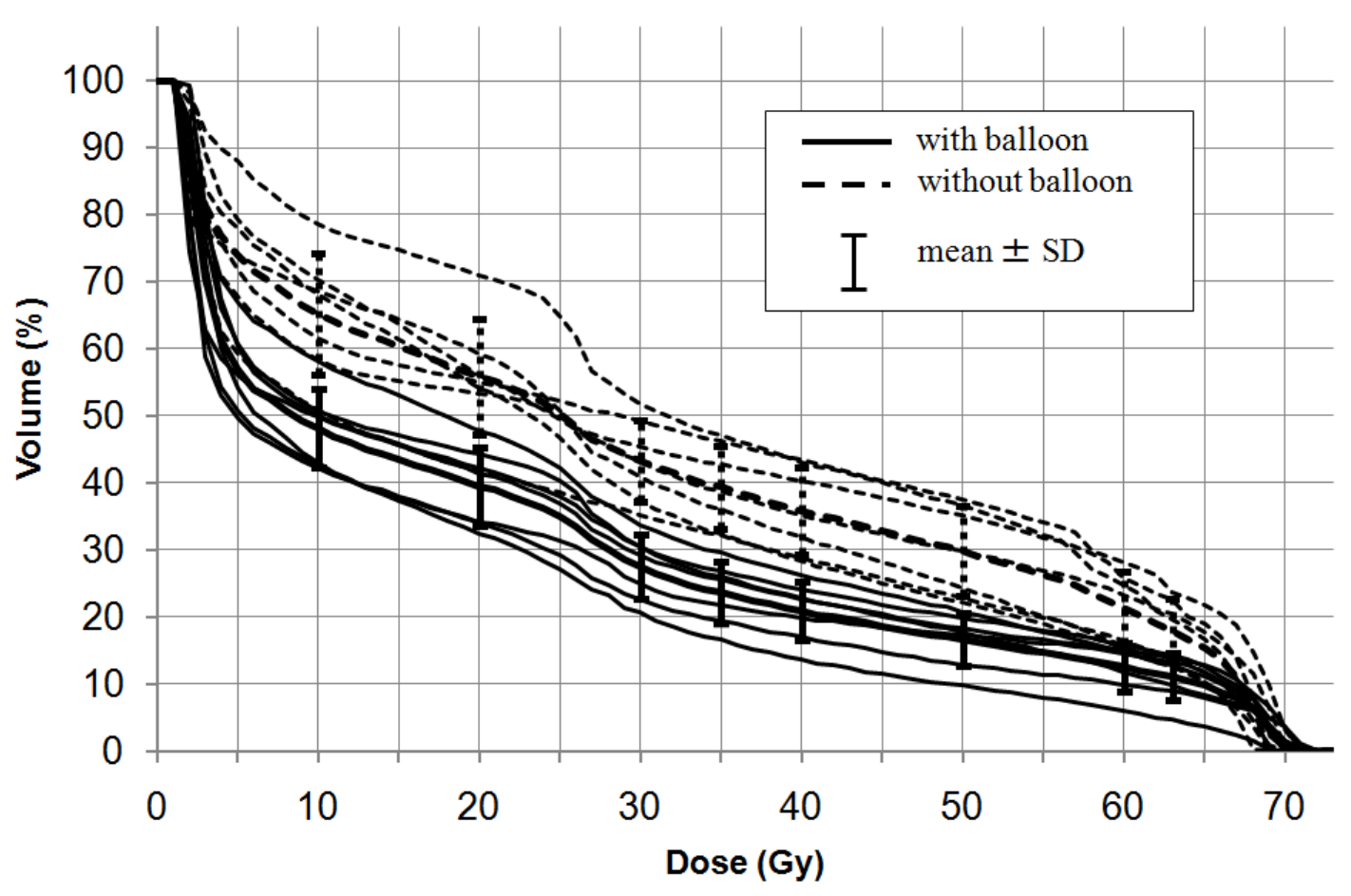

Figure 3: DVHs for the rectal wall and PTV

3.a: DVHs for the rectal wall, 3.b: DVHs for PTV

Each thin line shows a DVH of each patient. In the figure 3.a, each thick line shows the mean DVH of each group, and bars indicate 1 standard deviation of each group at the representative dose levels.

Abbreviation: DVH: dose volume histogram 


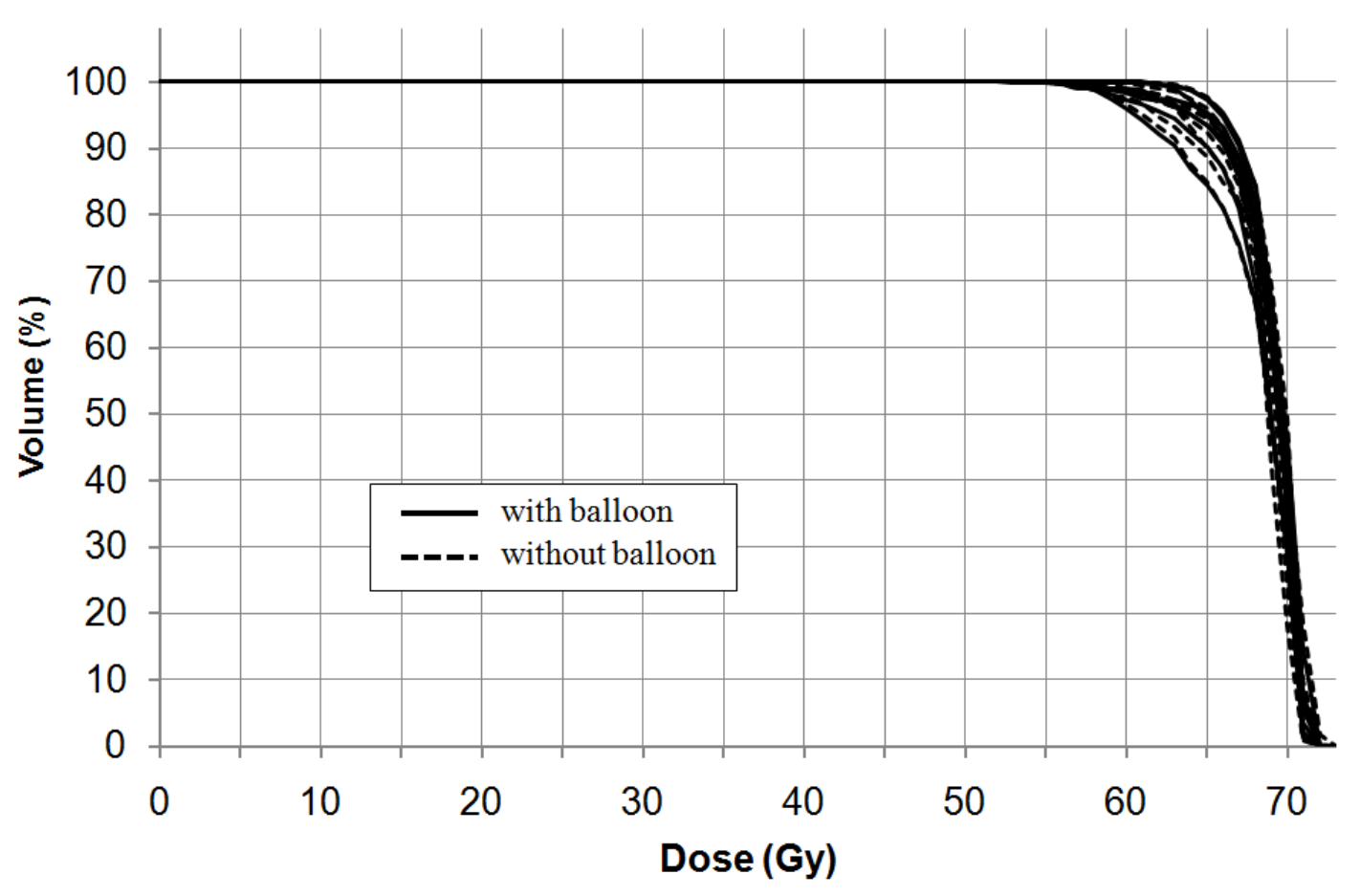

3.b: DVHs for PTV 


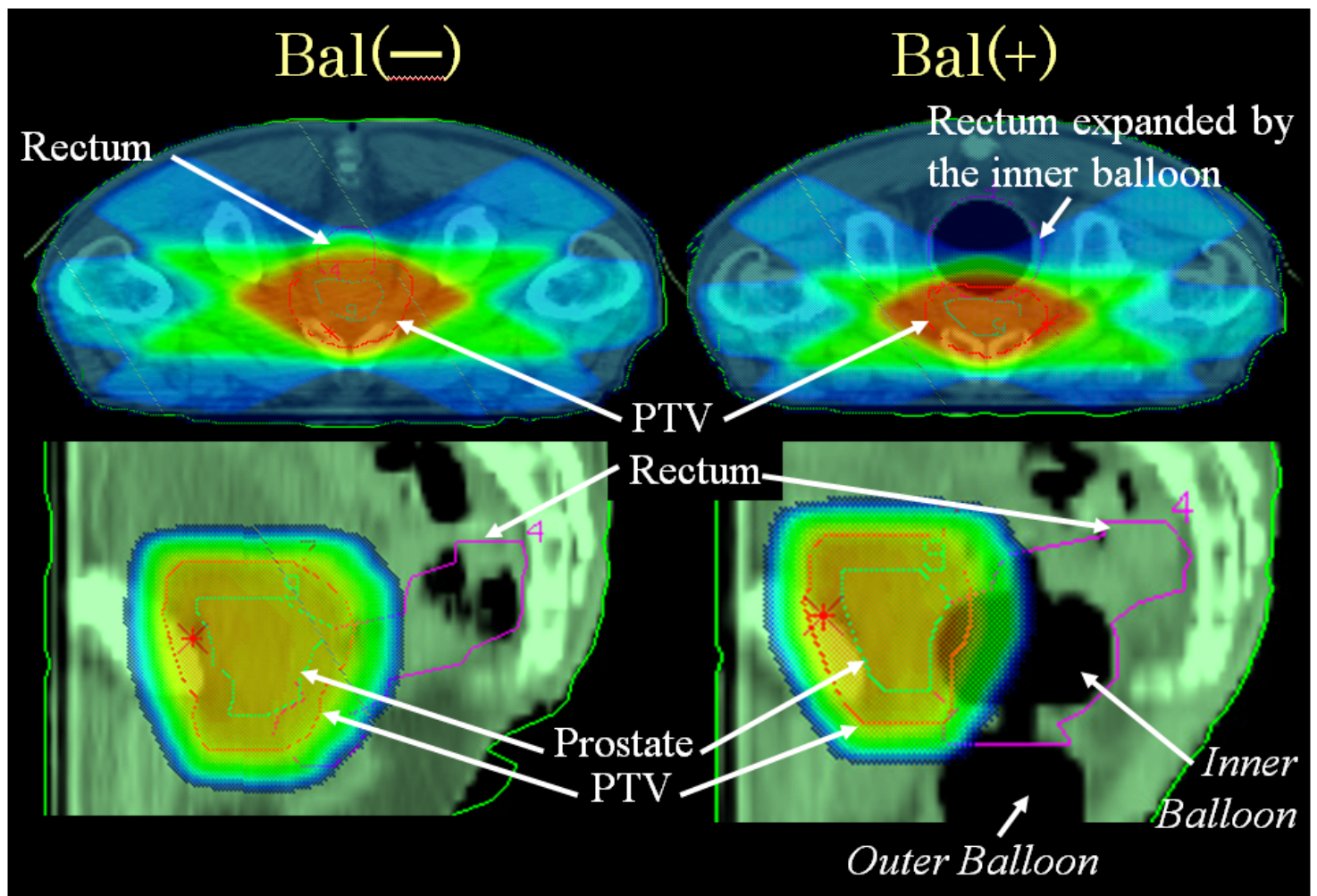

Figure 4: Dose distribution with or without a rectal balloon catheter

The inner balloon expands the rectum and prevents the posterior rectal wall from being included within the intermediate-dose area. 
Table 1: Comparison of $V_{35}, V_{50}$ and $V_{63}$ of the rectal wall

\begin{tabular}{lccc}
\hline & \multicolumn{2}{c}{ Mean \pm SD } & p-value \\
\cline { 2 - 3 } & With balloon & Without balloon & 0.0004 \\
\hline $\mathrm{V}_{35}$ & $23.7 \pm 4.6$ & $39.3 \pm 6.2$ & 0.002 \\
$\mathrm{~V}_{50}$ & $16.6 \pm 3.8$ & $29.8 \pm 6.7$ & 0.008 \\
$\mathrm{~V}_{63}$ & $11.1 \pm 3.5$ & $18.0 \pm 4.7$ & 0 \\
\hline
\end{tabular}

Abbreviation. $V_{35}, V_{50}$, and $V_{63}$ : the percent volumes of the rectal wall receiving 35,50 , and 63 Gy, respectively 
Table 2: The absolute value of the displacements

\begin{tabular}{c|cccc}
\hline \multicolumn{2}{c}{ Maximum } & Mean \pm SD & p-value \\
\hline $\mathrm{A}$ & Bal (+) & 3.0 & $1.3 \pm 0.9$ & 0.014 \\
$\mathrm{P}$ & Bal (-) & 6.0 & $2.8 \pm 1.8$ & \\
\hline $\mathrm{S}$ & Bal (+) & 4.0 & $1.4 \pm 1.4$ & n.s. \\
$\mathrm{I}$ & Bal (-) & 5.0 & $2.7 \pm 1.8$ & \\
\hline $\mathrm{L}$ & Bal (+) & 3.0 & $1.2 \pm 1.0$ & n.s. \\
$\mathrm{R}$ & Bal (-) & 3.0 & $1.3 \pm 0.7$ & \\
\hline 3 & Bal (+) & 5.8 & $2.6 \pm 1.4$ & 0.014 \\
$\mathrm{D}$ & Bal (-) & 7.9 & $4.5 \pm 1.8$ & \\
\hline
\end{tabular}

The unit is millimeter.

Abbreviation. AP: anterior-posterior, SI: superior-inferior, LR: left-right, 3D: three dimensional, Bal (+): with balloon, Bal (-): without balloon 
Table 3: Systematic \& random organ motion

\begin{tabular}{c|ccc}
\hline & & Systematic & Random \\
\multicolumn{1}{l}{} & & $($ Mean \pm SD) & $($ SD $)$ \\
\hline A & Bal (+) & $0.4 \pm 1.3$ & 0.9 \\
$\mathrm{P}$ & Bal (-) & $1.4 \pm 2.4$ & 1.9 \\
\hline $\mathrm{S}$ & Bal (+) & $-0.3 \pm 1.5$ & 1.4 \\
$\mathrm{I}$ & Bal (-) & $1.9 \pm 2.3$ & 1.5 \\
\hline $\mathrm{L}$ & Bal (+) & $-0.7 \pm 1.2$ & 0.8 \\
$\mathrm{R}$ & Bal (-) & $-0.2 \pm 1.4$ & 1.1 \\
\hline
\end{tabular}

The unit is millimeter. Negative values indicate posterior (dorsal), inferior (caudal) and right, respectively.

Abbreviation: same as Table 2 